\title{
Climatology of nighttime medium-scale traveling ionospheric disturbances (MSTIDs) in the Central Pacific and South American sectors
}

\author{
T. M. Duly, N. P. Chapagain, and J. J. Makela \\ Department of Electrical and Computer Engineering, University of Illinois at Urbana-Champaign, Urbana, IL, USA
}

Correspondence to: T. M. Duly (duly2@illinois.edu)

Received: 30 August 2013 - Revised: 1 November 2013 - Accepted: 7 December 2013 - Published: 20 December 2013

\begin{abstract}
We present occurrence rate statistics for nighttime medium-scale traveling ionospheric disturbances (MSTIDs) in the Central Pacific and South American sectors using data collected by $630.0 \mathrm{~nm}$ filtered CCD imaging systems. The data were collected from September 2006 through December 2012. In general, the statistics are in good agreement with the basic linear theory of MSTIDs, with observations coinciding with low F10.7A values, representative of solar minimum. Overall, MSTIDs are observed in approximately $68 \%$ of the usable nights near the solstices at mid-latitudes and approximately $20 \%$ of the usable nights for equinox periods. Observations closer to the geomagnetic equator yielded a maximum occurrence rate of about 10-20\% during the solstices and about $0-3 \%$ during the equinoxes. The lower number of MSTID observations near the low latitudes is attributed to limitations of MSTID growth rate, propagation, and/or geometrical observational effects. The relatively large number of MSTID occurrences during the solstices can be accounted for by the neutral wind contribution to the MSTID growth rate either at the local or magnetic conjugate point.
\end{abstract}

Keywords. Ionosphere (ionospheric disturbances; midlatitude ionosphere)

\section{Introduction}

Nighttime medium-scale traveling ionospheric disturbances (MSTIDs) are a phenomenon often detected in the midlatitude ionosphere characterized by waves of raised and lowered bands of electron density. They typically have wavelengths of the order of $100-500 \mathrm{~km}$ and travel at an angle to the magnetic field in the westward and equatorward directions at velocities ranging from 50 to $150 \mathrm{~m} \mathrm{~s}^{-1}$ (e.g.,
Garcia et al., 2000; Kelley, 2011). Radar experiments have verified that MSTIDs are polarized structures, with internal electric fields of the order of $5 \mathrm{mV} \mathrm{m}^{-1}$ (Behnke, 1979; Saito et al., 1995; Kelley et al., 2000; Shiokawa et al., 2003b). MSTIDs have been observed in conjugate hemispheres (e.g., Otsuka, 2004) due to the polarization electric field within an MSTID mapping efficiently along magnetic field lines, thereby creating a mirrored structure in the conjugate hemisphere.

One of the first detections of MSTIDs was by Behnke (1979) using the incoherent scatter radar at the Arecibo Observatory in Puerto Rico to make measurements of the height-band variations in the $\mathrm{F}$ region. The radar was operated in a mode in which measurements were taken from different azimuth angles, from which the orientation and propagation direction of the structures could be deduced. Behnke (1979) attributed these height-band variations to the Perkins instability (Perkins, 1973), in part due to the angle the propagating waves made with the magnetic field. In his model of the mid-latitude ionosphere, Perkins solved the momentum equations by integrating quantities along the magnetic field line and found the existence of unstable modes when a north-south electric field or eastern neutral wind existed (in the Northern Hemisphere configuration) (Perkins, 1973). The associated forces upset the balance of the mid-latitude ionosphere whose equilibrium is supported by the combination of an eastward electric field and southern neutral wind against gravity for regions containing a finite dip angle in the Northern Hemisphere. The instability sets up a polarized current density, which through an $\boldsymbol{E} \times \boldsymbol{B}$ drift creates the raised and lowered bands of electron density (Zhou and Mathews, 2006). 
There have been a few issues with invoking the Perkins instability as the generative mechanism for MSTIDs (e.g., Kelley et al., 2002b). Specifically, the growth rate is too small for the instability to grow by an appreciable amount from thermal noise alone. As such, it is generally accepted that some additional seed is required to augment the low Perkins growth rate to allow for the development of MSTIDs. Huang et al. (1994) identified gravity waves as a plausible explanation for driving MSTIDs in the bottomside $\mathrm{F}$ region, thus enhancing their growth rate. There is also a growing body of literature exploring $\mathrm{E}$ region coupling effects, through which the polarized electric field from a sporadic $E$ layer maps efficiently to the $F$ region, providing the seeding mechanism for MSTIDs (Tsunoda and Cosgrove, 2001; Cosgrove, 2004; Tsunoda, 2006; Yokoyama and Hysell, 2010).

Auroral radar experiments from the Super Dual Auroral Radar Network (SuperDARN) conducted by Ogawa et al. (2009) studied MSTIDs initiated as gravity waves. Using the results from this study, Kelley (2011) proposed that Joule heating dampens out every $\boldsymbol{k}$ vector except ones associated with the Perkins instability. The filtered modes are then amplified at mid-latitudes with the coupled $\mathrm{E}$ region dynamics (i.e., Tsunoda and Cosgrove, 2001), thus forming an MSTID strong enough to be observed.

Properties of MSTIDs have been studied through the combination of theory and data obtained from climatological studies. These studies aid the theoretical understanding of MSTIDs by providing statistical trends of their nature. Of particular interest for MSTID climatologies are their properties (i.e., wavelength, velocity, total electron content (TEC) fluctuations) and their seasonal and solar cycle dependence for developing and propagating in the nighttime ionosphere. For example, using electric field fluctuations measured by the Dynamic Explorer 2 satellite, Saito et al. (1995) compiled the first global statistical occurrence rates for MSTIDs over a 19-month period. They were able to verify matching electric field fluctuations between conjugate hemispheres, which lead to the theory of polarization electric fields within an MSTID map along the magnetic field lines to the conjugate hemisphere.

Otsuka et al. (2011) used a dense network of GPS receivers in Japan to study the TEC fluctuations characteristic of MSTIDs, in effect generating maps of 2-D spatial and temporal information. Similarly, single GPS receivers measuring TEC fluctuations have been used to build climatological databases for regions in Japan, Europe, the United States, Australia, and South America during 1998, 2000, and 2001 (Kotake et al., 2006). These studies have shown MSTIDs occurring during low solar flux conditions in the June and December solstices.

In addition, $630.0 \mathrm{~nm}$ filtered CCD imaging systems have been utilized to study MSTIDs via observations of the airglow emission caused by the dissociative recombination of $\mathrm{O}_{2}^{+}$. This reaction occurs predominantly in the bottomside nighttime $\mathrm{F}$ region where the relatively large population of
$\mathrm{O}_{2}^{+}$recombines, thereby producing photons with a $630.0 \mathrm{~nm}$ wavelength (Link and Cogger, 1988). The electron density altitude variations (characteristic of MSTIDs) are observed in airglow imagers as enhanced and depleted bands. Like the dense network of GPS receivers, airglow imaging systems provide 2-D spatial and temporal information on MSTIDs. For a review of imaging systems used to study the ionosphere, see Makela and Otsuka (2011).

With the aid of imaging systems, seasonal and solar cycle trends of MSTID occurrences have been studied in the Japanese sector (Shiokawa et al., 2003a), Eastern Asian sector (Fukushima et al., 2012), and also at Arecibo (Garcia et al., 2000; Martinis et al., 2010). Candido et al. (2008) have presented a statistical analysis of MSTID occurrences at $\mathrm{Ca}$ choeira Paulista, Brazil $\left(-22.7^{\circ} \mathrm{N}, 315.0^{\circ} \mathrm{E}\right.$, dip $\left.=-30.9^{\circ}\right)$ using $630.0 \mathrm{~nm}$ emission airglow images during local winter (June-July). These studies show a semiannual pattern with MSTID occurrence peaks during the solstice months, and have also illustrated that occurrence rates are inversely proportional to solar flux.

Here, we contribute to the climatological database pertaining to the occurrence statistics of MSTIDs using airglow imaging data collected from $630.0 \mathrm{~nm}$ filtered CCD imaging systems for two geographical sectors: Haleakala, Hawaii $\left(20.71^{\circ} \mathrm{N}, 203.74^{\circ} \mathrm{E}\right)$ in the Central Pacific and Cerro Tololo, Chile $\left(-30.17^{\circ} \mathrm{N}, 289.19^{\circ} \mathrm{E}\right)$ in the South American sector. This is the first long-term study of MSTIDs in the Central Pacific sector, filling the data gap between studies conducted in the Japanese and Brazilian sectors. The site at Cerro Tololo is northwest of the Martinis et al. (2006) study located in El Leoncito, Argentina, and provides observations on the west coast of South America. Observations at Haleakala in the Central Pacific sector are approximately $6000 \mathrm{~km}$ southeast of the sites used in the previously studied Japanese sector. Data from Haleakala and Cerro Tololo were selected from September 2006 through December 2012, allowing for coincident longitudinal comparisons to be made. In addition, the times associated with this long-term data set include recent solar minimum to solar moderate conditions, permitting an analysis of solar cycle dependence.

Section 2 describes the instrumentation used at the two locations, including the camera properties and associated viewing geometries. Section 3 reviews the methods used to analyze the data and presents the results of the climatology. A discussion follows in Sect. 4 analyzing the statistical results with respect to season, month, and solar flux values. Also, the results are compared with previous studies found in the literature. Conclusions are presented in Sect. 5.

\section{Instrumentation}

At each site, CCD cameras equipped with a $630.0 \mathrm{~nm}$ filter are used to gather statistical data on the occurrence rates of MSTIDs. At Haleakala, two imaging systems provide 


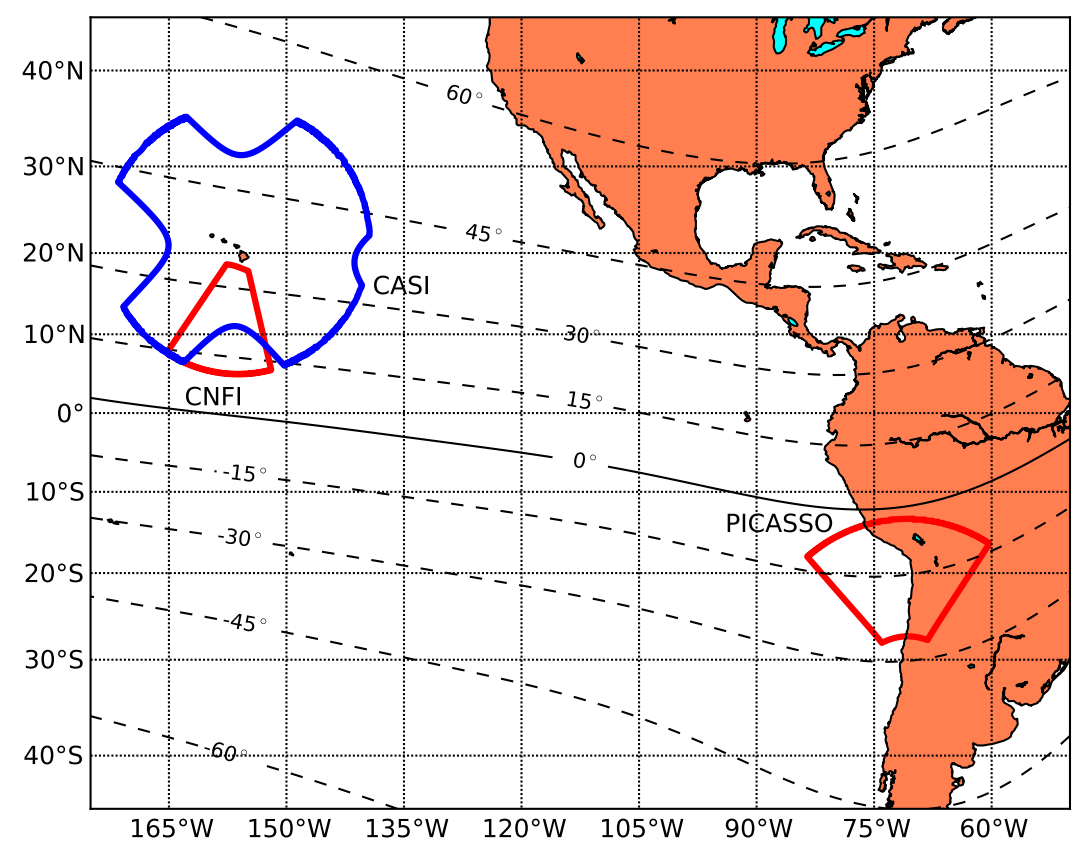

Fig. 1. Fields of view for CASI, CNFI, and PICASSO at an altitude of $250 \mathrm{~km}$. Also shown are magnetic dip angle values at $250 \mathrm{~km}$.

different spatial resolutions and coverages for the regions of interest. The cameras are operational at night and are controlled by software to cycle through various optical filters and to periodically record dark images that can be used in the analysis to reduce the effects of noise.

Figure 1 summarizes the viewing geometry of the instrumentation, showing the field of view of each instrument at an assumed airglow emission altitude of $250 \mathrm{~km}$. Also overlaid on Fig. 1 is a magnetic field dip angle contour map. The dip angles were obtained using the eleventh-generation International Geomagnetic Reference Field (IGRF11) model (Finlay et al., 2010) for a year input of 2010 and an altitude of $250 \mathrm{~km}$.

\subsection{Haleakala, Hawaii}

Two imaging systems, each with different viewing geometries, are co-located at the Haleakala Volcano on the island of Maui, Hawaii $\left(20.71^{\circ} \mathrm{N}, 203.74^{\circ} \mathrm{E}\right)$. Both the Cornell All-Sky Imager (CASI) and Cornell Narrow-Field Imager (CNFI) employ a $1024 \times 1024$ pixel CCD array binned to $512 \times 512$ pixels. Thermal noise is reduced by cooling each imager to $-40^{\circ} \mathrm{C}$. The $630.0 \mathrm{~nm}$ filtered images are made using an exposure time of $90 \mathrm{~s}$. In addition to studying the properties of MSTIDs, CASI and CNFI have been used extensively for studying equatorial plasma bubbles (EPBs) since the early 2000s (e.g., Kelley, 2002a; Makela et al., 2004). In the current study, data are obtained beginning in September 2006 to coincide with observations from Cerro Tololo, Chile.
CASI has a center look direction at zenith and a $180^{\circ}$ field of view. CNFI has a field of view of $47^{\circ}$ and looks south with an azimuth angle of $188.0^{\circ}$ and an elevation angle of $17.9^{\circ}$. The viewing configuration of CNFI was chosen such that the field of view is along the magnetic field lines in the airglow layer, enabling an optimal viewing configuration of EPBs as originally proposed by Tinsley (1982). Although field-aligned irregularities such as EPBs were not of primary interest in this study, the viewing configuration of CNFI enables MSTID detection for regions equatorward of Hawaii at dip angles ranging from $15^{\circ}$ to $30^{\circ}$. Also, this viewing configuration gives higher spatial resolutions for lower elevation angles than the CASI system, allowing for clearer detection of MSTIDs at lower latitudes.

\subsection{Cerro Tololo, Chile}

A Portable Ionospheric Camera and Small-Scale Observatory (PICASSO) is located at the Cerro Tololo InterAmerican Observatory (CTIO) near La Serena, Chile $\left(-30.17^{\circ} \mathrm{N}, 289.19^{\circ} \mathrm{E}\right)$. This narrow-field imager is similar to CNFI, as it looks equatorward along the magnetic field lines to reduce blurring effects for observing FAIs. PICASSO's field of view covers dip angles from approximately $-20^{\circ}$ to $0^{\circ}$.

PICASSO is equipped with a $-30^{\circ} \mathrm{C}$ cooled $2184 \times 1472$ CCD array (binned by a factor of 3 ) and records 90 s exposures of the $630.0 \mathrm{~nm}$ emission. The field of view of this imager is approximately $80^{\circ} \times 60^{\circ}$, covering approximately $18^{\circ}$ of geomagnetic latitude at an assumed emission altitude of $250 \mathrm{~km}$. PICASSO has been used in previous studies to 

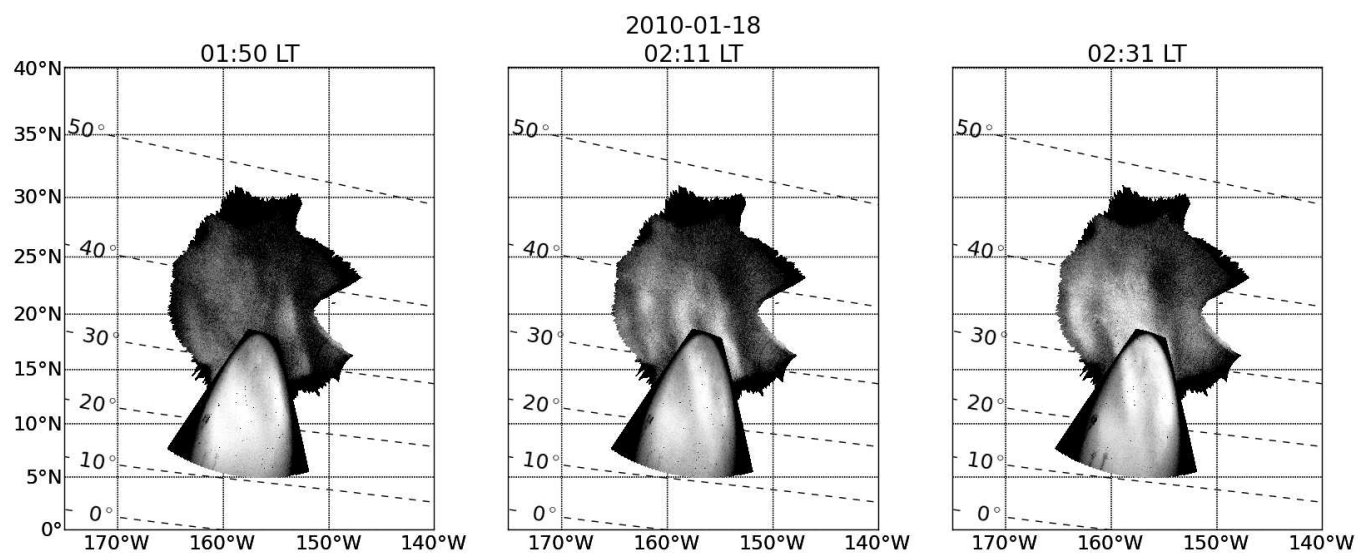

Fig. 2. An example image sequence displaying an MSTID passing through the fields of view of CASI and CNFI. Also shown are magnetic dip angles at $250 \mathrm{~km}$.

observe MSTIDs at low geomagnetic latitudes (e.g., Makela et al., 2010). This instrument has been providing MSTID observations since September 2006.

\section{Methodology}

To formulate the climatology, we hand-classify each night's images with labels of "usable", "MSTID", and "cloudy/bad data". The subset of "MSTID" labels was included in the set of "usable" labels, as usable nights consisted of clear skies with the possibility of observing ionospheric structure. In other words, a night with label "MSTID" was also labeled "usable". An MSTID was determined to be present in the data by recognizing the distinct bands traveling westward and toward the Equator (however, it should be noted that one MSTID observed by CASI was found to travel east and northward and was accounted for in the statistics with an MSTID label). Bad data correspond to nights when the instrument experienced light contamination (e.g., when the moon was up) or when the instrument malfunctioned.

MSTID candidates were verified against false positives by using imaging data filtered for different emissions (i.e., the $557.7 \mathrm{~nm}$ green line and the $777.4 \mathrm{~nm}$ near IR). MSTIDs are not typically observed in images of these wavelengths. For example, the radiative recombination of $\mathrm{O}^{+}$(Tinsley et al., 1973) produces the $777.4 \mathrm{~nm}$ emission and is relatively weak (especially during the low solar conditions of the current study). Given that MSTIDs have TEC perturbations of the order of 1 TECU (Otsuka et al., 2011), which is below the sensitivity of our imaging systems at this wavelength, MSTIDs are not easily observed in the $777.4 \mathrm{~nm}$ emission line.

An example image sequence of MSTIDs observed over Hawaii is shown in Fig. 2. The images are approximately 20 min apart and show the NW-SE wavefronts of the MSTID traveling SW, which are detected in the fields of view of both CASI and CNFI.
We then collected the data labels into seasonal bins for each year, with December solstice months corresponding to November, December, January, and February; June solstice months corresponding to May, June, July, and August; and finally equinox months corresponding to March, April, September, and October. The MSTID occurrences were also binned monthly across all years to study monthly trends. Finally, the occurrences were binned as a function of associated 81-day averaged solar flux (F10.7A) to study their solar cycle dependence.

Data were analyzed for all three instruments from September 2006 through December 2012. From the instruments in the Central Pacific, we processed 820 and 1331 usable nights for CASI and CNFI, respectively. Data from Cerro Tololo totaled 939 usable nights. Figures 3, 4, and 5 display the summary statistics for CASI, CNFI, and PICASSO, respectively. In each figure, the left-hand plots display the seasonal statistics for the December solstice, June solstice, and equinox periods. The top right plot of each figure displays data binned into individual months, while the lower right plot shows the data binned by F10.7A value. In each plot, the left-hand ordinate corresponds to the raw counts for the usable nights (wide red bars) and MSTIDs (thin cyan bars). The right-hand ordinate displays the corresponding occurrence rate (blue dashed line), which is the ratio of MSTID counts to usable night counts.

\section{Discussion}

Overall, from the 820 usable nights observed by CASI, $48.9 \%$ showed occurrences of MSTIDs. CNFI recorded an occurrence rate of $7.0 \%$ from 1331 usable nights. Finally, PICASSO recorded the smallest rate of occurrences, with $4.7 \%$ from 939 usable nights. 


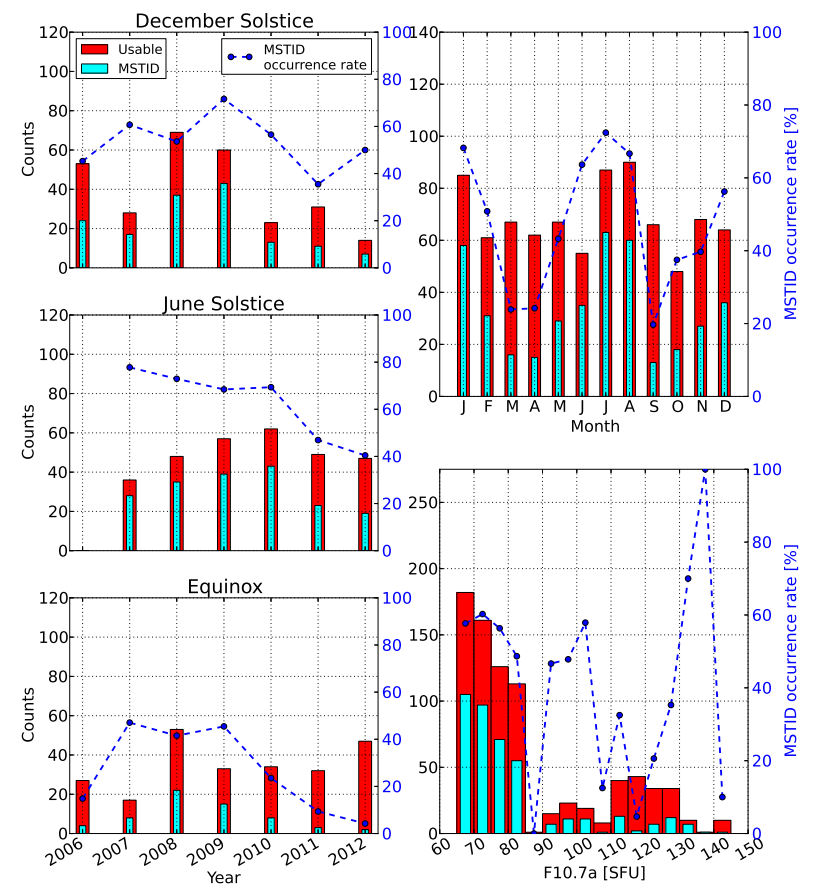

Fig. 3. Summary statistics for CASI (see text for discussion).

Although relatively few, the MSTIDs observed by CNFI and PICASSO are significant because they are observed at such low geomagnetic latitudes. Previous studies of MSTIDs have hypothesized the equatorward limit of their propagation to be around $18^{\circ}$ magnetic latitude (Shiokawa et al., 2002). However, the low latitudinal extent of MSTIDs has been shown before (Makela et al., 2010), and in the current study, several instances of MSTIDs were observed propagating toward the geomagnetic equator at magnetic latitudes well equatorward of $18^{\circ}$. A further quantitative analysis of MSTID propagation limits within the current data set is required to study the hypothesized equatorward limitation.

An interesting feature of the summary statistics is the prevalence of MSTIDs observed from CASI compared with CNFI and PICASSO. The low elevation viewing geometry of CNFI and PICASSO could inhibit the detection of MSTIDs, as the look directions of these instruments integrate through MSTID wavefronts. Another plausible explanation for the occurrence difference is due to the growth rate of MSTIDs being proportional to $\sin ^{2} D$, where $D$ is the magnetic inclination angle (Perkins, 1973; Hamza, 1999). The fields of view of CNFI and PICASSO encompass smaller inclination angles, thus the growth rate, and consequently the occurrence rate is smaller at these locations. Finally, MSTIDs could be subject to propagation limitations as they travel equatorward. For example, given an MSTID observed by CASI, the structure may not propagate far enough to the south to be viewed by CNFI before local sunrise. For the structures that successfully propagate into CNFI's field of view, the MSTIDs are

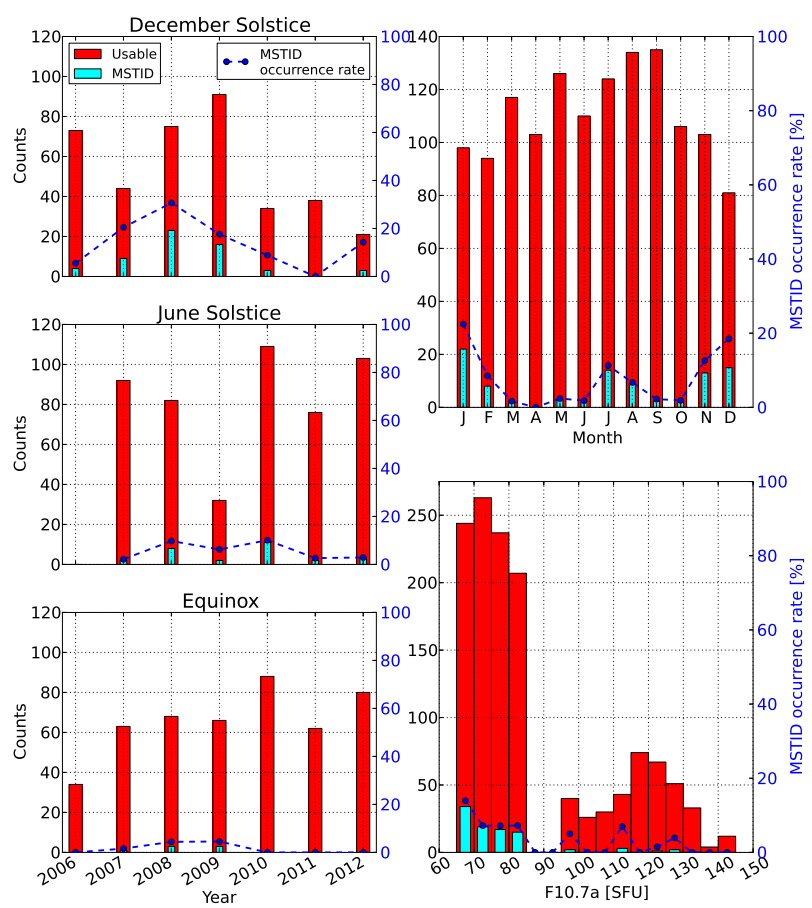

Fig. 4. Summary statistics for CNFI.

often observed from CASI's field of view about 30-90 min prior, therefore making MSTIDs observed from CNFI an approximate subset of MSTIDs observed from CASI. The statistics show that MSTIDs observed in CASI's field of view are not always detected in CNFI's field of view, limited by growth rate, propagation, and/or geometrical observation effects.

Solstice and equinox occurrence trends of MSTIDs can be established with the aid of the binned seasonal plots (the lefthand plots of Figs. 3-5). For the December solstice, CASI recorded relatively high occurrence rates ranging between $35 \%$ and $72 \%$ throughout the years of observation. Comparatively, CNFI and PICASSO recorded fewer observations for the December solstice, possibly due to the low geomagnetic dip angle viewing geometry of these instruments as previously discussed. CNFI recorded a maximum of 23 observations ( $30.7 \%$ occurrence rate) in 2008 for the December solstice time period, while PICASSO had a maximum of 7 counts $(11.7 \%$ occurrence rate) in 2008 for the same time period.

During the June solstice, CASI recorded high MSTID counts, with occurrence rates ranging between $40 \%$ and $78 \%$. With respect to CASI, fewer MSTID occurrences were recorded by CNFI and PICASSO. CNFI observed a maximum of 11 counts ( $10.1 \%$ occurrence rate) in 2010 during the June solstice period and PICASSO recorded no more than three observations in a given year. 


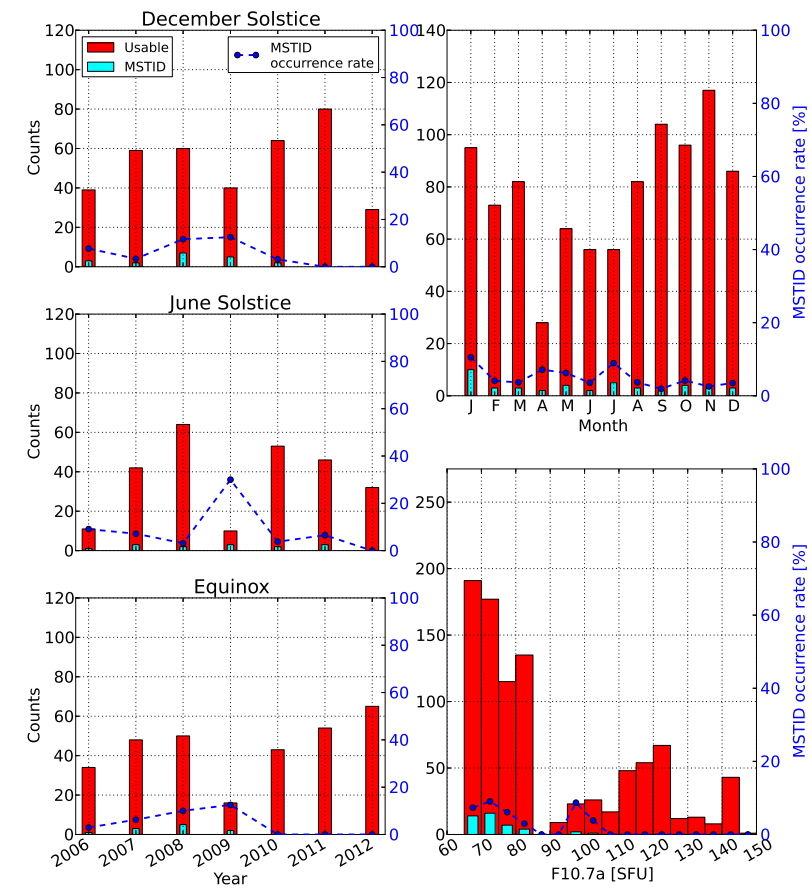

Fig. 5. Summary statistics for PICASSO.

Similar to the other time periods, greater MSTID counts were recorded by CASI during the equinox period compared with CNFI and PICASSO. CASI recorded as many as 22 counts $(41.5 \%$ occurrence rate) during the equinox period for 2008. CNFI recorded only [0,1,3,3] MSTID counts in [2006, 2007, 2008, 2009] during the equinox period with a maximum occurrence rate of $4.5 \%$ in 2009. PICASSO had [1,3, 5, 2] MSTID counts in years [2006, 2007, 2008, 2009] for the equinox time frame with a maximum occurrence rate of $12.5 \%$ in 2009 . However, only 16 usable nights were available during the 2009 equinox period, perhaps biasing the occurrence rate.

In general, MSTIDs from this data set are observed primarily during the solstices. When binned by months, data from each of the three instruments show peaks in the occurrence rate near the June and December solstices. It is difficult to determine the major and minor peaks of these data, as sampling biases may influence the occurrence rate. For example, the monthly binned data for CNFI (upper right plot of Fig. 4) appear to have a major peak in the occurrence rate near the December solstice, but there were also fewer usable night counts in the December solstice compared with the June solstice time frame.

The solstice occurrence peaks of MSTIDs from the current study could be attributed to the neutral wind component of the maximum growth rate. Following Garcia et al. (2000), the neutral wind contribution to the maximum growth rate for MSTIDs can be expressed in the form:

$\gamma_{\max } \propto u \sin D \sin \delta+u \cos \delta-v \sin \delta-v \sin D \cos \delta$,

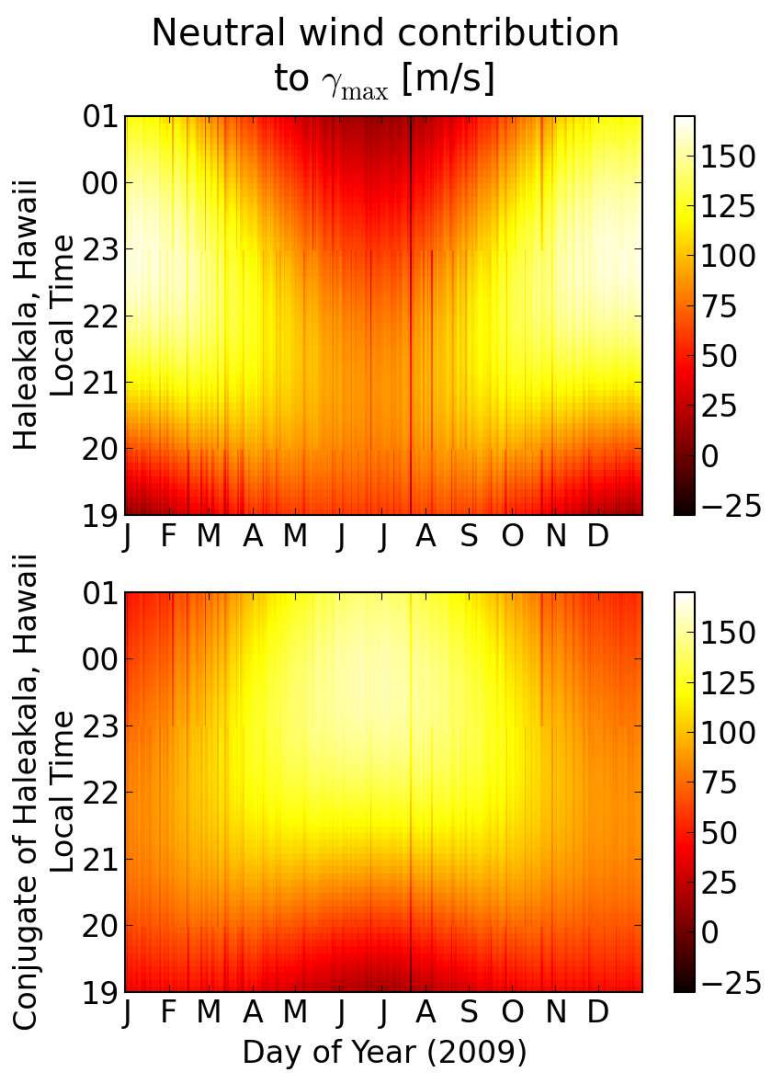

Fig. 6. The neutral wind contribution to $\gamma_{\max }$ (Eq. 1) plotted as a function of local time and day of year for Haleakala (top) and the magnetic conjugate point of Haleakala (bottom).

where $D$ is the magnetic inclination (dip) angle, $\delta$ is the magnetic declination angle, and $u$ and $v$ are the zonal (positive eastward) and meridional (positive northward) neutral winds, respectively. Equation (1) is plotted in Fig. 6 for Haleakala (top) and the geomagnetic conjugate point of Haleakala (bottom) as a function of local time and day of year for 2009 (using other years from this study yielded similar trends as Fig. 6). For these plots, $u$ and $v$ are obtained from the horizontal wind model HWM93 (Hedin et al., 1996) and the inclination and declination angles are from IGRF (Finlay et al., 2010). The quantities $u$ and $v$ provided by HWM93 are not exact measurements, but represent the general climatology of the neutral wind for a given time. The climatological values are sufficient to elucidate the general seasonalities of the neutral wind contribution to the maximum growth rate of MSTIDs given in Eq. (1).

The top plot of Fig. 6 shows that for Haleakala, Eq. (1) is largest near pre-midnight times during the December solstice period. On the other hand, at the conjugate point of Haleakala, Eq. (1) is largest near pre-midnight hours during the June solstice period. It is likely that the December solstice occurrence peaks at Haleakala are due to the conducive neutral winds contributing to the maximum growth 
rate for the development of MSTIDs, and that the June solstice occurrence peaks are due to MSTIDs being generated in the conjugate hemisphere during those time frames and efficiently mapping to the Haleakala location. The observations of MSTIDs from this study were predominantly in the premidnight time frame, which is in good agreement with the large contribution to the growth rate given in Fig. 6 during these times.

The data selected from these sites encompass the recent deep solar minimum time frame, providing insight into the occurrence of MSTIDs at low F10.7A values. Comparing all three instruments, we observed MSTIDs primarily during low solar flux values, representative of solar minimum. In fact, CASI recorded 105 counts of MSTID occurrences (from a possible 182 usable nights) with an F10.7A value less than 70 SFU. Very few MSTID observations were recorded by CNFI and PICASSO for days with a solar flux value greater than $90 \mathrm{SFU}$. The Perkins growth rate is inversely proportional to $\left\langle v_{\text {in }}\right\rangle$, the plasma density-weighted average value of the ion-neutral collision frequency (Perkins, 1973; Kelley and Fukao, 1991). Ion density (and consequently $\left\langle\nu_{\text {in }}\right\rangle$ ) is relatively low at solar minimum, and therefore the theoretical growth rate is higher. From the data set of the current study, the observations of MSTIDs during solar minimum agree with this description.

To compare the occurrences of MSTIDs at two geographical sectors, results from CNFI are selected to compare with PICASSO as both instruments look equatorward and toward low geomagnetic latitudes, thus removing any major biasing in the occurrence rates caused by latitudinal and observational/geometrical effects. The general trend of occurrence peaks near the solstices is prevalent, but PICASSO only had an occurrence rate of $10.5 \%$ and $8.9 \%$ for January and July, respectively, while CNFI had higher rates of $22.4 \%$ and $11.3 \%$ for these months. Although both CNFI and PICASSO look at low geomagnetic latitudes, the minor discrepancies of viewing geometry between the instruments may be responsible for the differences in occurrence rates. Figure 1 shows that the viewing geometry of PICASSO is slightly more equatorward compared with CNFI, therefore MSTID development observed by PICASSO could be subject to the greater geomagnetic latitude restrictions previously discussed.

We now compare these occurrence statistics with studies found in the literature. At Arecibo $\left(18.34^{\circ} \mathrm{N}, 293.25^{\circ} \mathrm{E}\right.$, $\operatorname{dip}=46.5^{\circ}$ ), Garcia et al. (2000) used an all-sky imager to observe MSTIDs and found a peak in the December solstice time frame (unfortunately, data from the June solstice were unavailable). This is consistent with our results of occurrence peaks for the December solstice time frames for all three instruments.

In the Japanese sector, Shiokawa et al. (2003a) used allsky $630.0 \mathrm{~nm}$ imagers to develop statistics of the occurrence of MSTIDs. They found a major maximum in the occurrence peak of MSTIDs near the June solstice of about $55 \%$ for Rikubetsu, Japan $\left(43.5^{\circ} \mathrm{N}, 143.8^{\circ} \mathrm{E}\right.$, dip $\left.=57.4^{\circ}\right)$. This rate is slightly lower than our major peak rate for CASI ranging from $64 \%-72 \%$ for June and July (compare our Fig. 3 with Fig. 4 of Shiokawa et al., 2003a). In their study, Shiokawa et al. (2003a) found a lower occurrence rate for Shigaraki, Japan $\left(34.8^{\circ} \mathrm{N}, 136.1^{\circ} \mathrm{E}\right.$, dip $\left.=48.5^{\circ}\right)$ (which is south of Rikubetsu) of about $45 \%$ for times near the June solstice. Analyzing the December time frames, observations at Rikubetsu and Shigaraki yielded about $18 \%$ and $12 \%$ occurrence rates, respectively. Our data for CASI indicate a $56 \%$ MSTID occurrence rate for December, which is higher than the Japanese sites. It should be noted that the Shiokawa et al. (2003a) study was from October 1998 through September 2000, when F10.7A values ranged from $\sim 125 \mathrm{SFU}$ (March 1999) to 200 SFU (June 2000), whereas in this study the solar conditions ranged from $\sim 70$ SFU (November 2008) to $\sim 145 \mathrm{SFU}$ (October 2011). The different time periods may be responsible for the lower occurrences of MSTIDs in the Shiokawa et al. (2003a) study, as the solar conditions were higher than the current study. Although the magnitude of the occurrence peak values are different, the general trend of June and December solstice MSTID occurrence peaks appears to be consistent.

Another feature of our data is the relatively low occurrence rates recorded in Cerro Tololo, Chile, compared with the instrumentation in Hawaii. Low occurrence rates were also found by Martinis et al. (2006) in El Leoncito, Argentina $\left(-31.8^{\circ} \mathrm{N}, 290.7^{\circ} \mathrm{E}, \mathrm{dip}=-31.5^{\circ}\right)$, which is approximately $230 \mathrm{~km}$ southeast of Cerro Tololo. Analogous to CNFI and CASI located in Hawaii, the narrow field view of PICASSO observes lower geomagnetic latitudes compared to the allsky imager used in the Martinis et al. (2006) study. In their study, Martinis et al. (2006) observed MSTIDs in less than $5 \%$ of the nights between 2000 and 2005. Our complete data set from PICASSO includes 44 MSTID counts out of 939 usable nights, which gives a total occurrence rate of $4.7 \%$.

The study by Martinis et al. (2006) was performed primarily during solar maximum with the F10.7A reaching a maximum value of $\sim 220 \mathrm{SFU}$ in October 2001. Due to these solar conditions, it is expected that the occurrence rate would be relatively low. Solar conditions from our study ranged from solar minimum to moderate conditions, and it is surprising that low occurrence rates were also recorded from PICASSO. However, the equatorward viewing geometry for PICASSO may be responsible for the low occurrence rates. As previously mentioned, MSTIDs could be inhibited from being observed at these low geomagnetic latitudes. This constraint was observed at Hawaii where instances of MSTIDs detected by CASI were not observed to propagate into CNFI's field of view. It is unclear if MSTIDs south of PICASSO are restricted from propagating into the field of view of the imager or if they are even initially present.

In the Indonesian sector, Fukushima et al. (2012) conducted a study of MSTID occurrences at Kototabang, Indonesia $\left(-0.2^{\circ} \mathrm{N}, 100.3^{\circ} \mathrm{E}, \operatorname{dip}=-19.9^{\circ}\right)$ from 
October 2002 through October 2009. This region is located in the equatorial anomaly, analogous to the regions observed by CNFI and PICASSO but for a different longitudinal sector. The Fukushima et al. (2012) study encompassed a wide range of solar activity with $\mathrm{F} 10.7 \mathrm{~A}$ values ranging from $\sim 65 \mathrm{SFU}$ (November 2008) to $\sim 175 \mathrm{SFU}$ (October 2002). Their occurrence rate was proportional to solar flux values (see Fig. 3 of Fukushima et al., 2012) with a maximum occurrence rate of about $47 \%$ in 2004. They attributed the prevalence of MSTIDs during high solar flux periods to gravity wave activity. However, these occurrence rate data are inconsistent with our findings of MSTID occurrences being inversely proportional to solar activity. The instabilities observed in the Fukushima et al. (2012) study had east-west wavefronts propagating poleward. Given that the observational characteristics and occurrence rate data do not match the current study, perhaps the fundamental instability observed by Fukushima et al. (2012) is different from MSTIDs typically observed in the mid-latitude ionosphere.

\section{Conclusion}

In this study, we analyzed the seasonal and solar activity variations of MSTID occurrence rates for two longitudinal sectors at Haleakala, Hawaii and Cerro Tololo, Chile. This longterm study included times within the recent deep solar minimum and extended to solar-moderate conditions. The major findings of this study are:

1. There were fewer observations of MSTIDs recorded by CNFI and PICASSO with respect to CASI. The different viewing geometries of CNFI and PICASSO may be responsible for the lower occurrence rates. Both of these instruments look equatorward, where MSTID development may be subject to restrictions on growth rate and propagation. In addition, geometrical effects could decrease the number of observations obtained from CNFI and PICASSO.

2. Consistent with studies found in the literature, we found December and June solstice occurrence rate peaks prevalent at all three locations. At Haleakala, the occurrence peaks in the December solstice were attributed to the strong neutral winds at the location contributing to the growth rate. The June solstice observations were attributed to the development of MSTIDs at the conjugate location, which results in efficient mapping into the Northern Hemisphere.

3. The occurrence of MSTIDs in our data set was found to be inversely proportional to the F10.7A index. We attribute this to the growth rate of MSTIDs being inversely proportional to $\left\langle v_{\text {in }}\right\rangle$.
Acknowledgements. Airglow observations on Mount Haleakala are supported by the Air Force Office of Scientific Research under contract FA9550-05-0160494 to Cornell University as well as by the National Science Foundation through grant ATM-06-044654 to the University of Illinois. The continued operation of the instrument would not be possible without the on-site support of Jacob Burger. We thank Professor Michael C. Kelley for making the CASI and CNFI data available for our use. Work at the University of Illinois is supported by the National Science Foundation through grant ATM06-044654. N. P. Chapagain was supported by the NASA LWS Heliophysics Postdoctoral Fellowship Program.

Topical Editor K. Hosokawa thanks two anonymous referees for their help in evaluating this paper.

\section{References}

Behnke, R.: F layer height bands in the nocturnal ionosphere over Arecibo, J. Geophys. Res., 84, 974-978, doi:10.1029/JA084iA03p00974, 1979.

Candido, C. M. N., Pimenta, A. A., Bittencourt, J. A., and BeckerGuedes, F.: Statistical analysis of the occurrence of mediumscale traveling ionospheric disturbances over Brazilian low latitudes using OI $630.0 \mathrm{~nm}$ emission all-sky images, Geophys. Res. Lett., 35, L17105, doi:10.1029/2008GL035043, 2008.

Cosgrove, R. B.: Coupling of the Perkins instability and the sporadic $E$ layer instability derived from physical arguments, J. Geophys. Res., 109, A06301, doi:10.1029/2003JA010295, 2004.

Finlay, C. C., Maus, S., Beggan, C. D., Bondar, T. N., Chambodut, A., Chernova, T. a., Chulliat, A., Golovkov, V. P., Hamilton, B., Hamoudi, M., Holme, R., Hulot, G., Kuang, W., Langlais, B., Lesur, V., Lowes, F. J., Lühr, H., Macmillan, S., Mandea, M., McLean, S., Manoj, C., Menvielle, M., Michaelis, I., Olsen, N., Rauberg, J., Rother, M., Sabaka, T. J., Tangborn, A., Tø ffner Clausen, L., Thébault, E., Thomson, A. W. P., Wardinski, I., Wei, Z., and Zvereva, T. I.: International Geomagnetic Reference Field: the eleventh generation, Geophys. J. Int., 183, 1216-1230, doi:10.1111/j.1365-246X.2010.04804.x, 2010.

Fukushima, D., Shiokawa, K., Otsuka, Y., and Ogawa, T.: Observation of equatorial nighttime medium-scale traveling ionospheric disturbances in 630-nm airglow images over 7 years, J. Geophys. Res., 117, A10324, doi:10.1029/2012JA017758, 2012.

Garcia, F. J., Kelley, M. C., Makela, J. J., and Huang, C.-S.: Airglow observations of mesoscale low-velocity traveling ionospheric disturbances at midlatitudes, J. Geophys. Res., 105, 18407, doi:10.1029/1999JA000305, 2000.

Hamza, A. M.: Perkins instability revisited, J. Geophys. Res., 104, 22567, doi:10.1029/1999JA900307, 1999.

Hedin, A., Fleming, E., Manson, A., Schmidlin, F., Avery, S., Clark, R., Franke, S., Fraser, G., Tsuda, T., Vial, F., and Vincent, R.: Empirical wind model for the upper, middle and lower atmosphere, J. Atmos. Terr. Phys., 58, 1421-1447, doi:10.1016/00219169(95)00122-0, 1996.

Huang, C.-S., Miller, C. A., and Kelley, M. C.: Basic properties and gravity wave initiation of the midlatitude $\mathrm{F}$ region instability, Radio Science, 29, 395-405, doi:10.1029/93RS01669, 1994.

Kelley, M. C.: On the origin of mesoscale TIDs at midlatitudes, Ann. Geophys., 29, 361-366, doi:10.5194/angeo-29-361-2011, 2011. 
Kelley, M. C. and Fukao, S.: Turbulent upwelling of the midlatitude ionosphere: 2 . Theoretical framework, J. Geophys. Res., 96, 3747, doi:10.1029/90JA02252, 1991.

Kelley, M. C., Makela, J. J., Saito, A., Aponte, N., Sulzer, M., and González, S. A.: On the electrical structure of airglow depletion/Height layer bands over Arecibo, Geophys. Res. Lett., 27, 2837, doi:10.1029/2000GL000024, 2000.

Kelley, M. C., Makela, J. J., Ledvina, B. M., and Kintner, P. M.: Observations of equatorial spread-F from Haleakala, Hawaii, Geophys. Res. Lett., 29, 2003, doi:10.1029/2002GL015509, 2002a.

Kelley, M. C., Makela, J., and Saito, A.: The mid-latitude F region at the mesoscale: some progress at last, J. Atmos. Sol.-Terr. Phys., 64, 1525-1529, doi:10.1016/S1364-6826(02)00090-1, 2002b.

Kotake, N., Otsuka, Y., Tsugawa, T., Ogawa, T., and Saito, A.: Climatological study of GPS total electron content variations caused by medium-scale traveling ionospheric disturbances, J. Geophys. Res., 111, A04306, doi:10.1029/2005JA011418, 2006.

Link, R. and Cogger, L. L.: A reexamination of the O I 6300-Ånightglow, J. Geophys. Res., 93, 9883, doi:10.1029/JA093iA09p09883, 1988.

Makela, J. J. and Otsuka, Y.: Overview of Nighttime Ionospheric Instabilities at Low- and Mid-Latitudes: Coupling Aspects Resulting in Structuring at the Mesoscale, Space Sci. Rev., 168, 419-440, doi:10.1007/s11214-011-9816-6, 2011.

Makela, J. J., Ledvina, B. M., Kelley, M. C., and Kintner, P. M.: Analysis of the seasonal variations of equatorial plasma bubble occurrence observed from Haleakala, Hawaii, Ann. Geophys., 22, 3109-3121, doi:10.5194/angeo-22-3109-2004, 2004.

Makela, J. J., Miller, E. S., and Talaat, E. R.: Nighttime medium-scale traveling ionospheric disturbances at low geomagnetic latitudes, Geophys. Res. Lett., 37, L24104, doi:10.1029/2010GL045922, 2010.

Martinis, C., Baumgardner, J., Smith, S. M., Colerico, M., and Mendillo, M.: Imaging science at El Leoncito, Argentina, Ann. Geophys., 24, 1375-1385, doi:10.5194/angeo-24-13752006, 2006.

Martinis, C., Baumgardner, J., Wroten, J., and Mendillo, M.: Seasonal dependence of MSTIDs obtained from $630.0 \mathrm{~nm}$ airglow imaging at Arecibo, Geophys. Res. Lett., 37, L11103, doi:10.1029/2010GL043569, 2010.

Ogawa, T., Nishitani, N., Otsuka, Y., Shiokawa, K., Tsugawa, T., and Hosokawa, K.: Medium-scale traveling ionospheric disturbances observed with the SuperDARN Hokkaido radar, allsky imager, and GPS network and their relation to concurrent sporadic E irregularities, J. Geophys. Res., 114, A03316, doi:10.1029/2008JA013893, 2009.

Otsuka, Y.: Geomagnetic conjugate observations of mediumscale traveling ionospheric disturbances at midlatitude using all-sky airglow imagers, Geophys. Res. Lett., 31, L15803, doi:10.1029/2004GL020262, 2004.
Otsuka, Y., Kotake, N., Shiokawa, K., Ogawa, T., Tsugawa, T., and Saito, A.: Aeronomy of the Earth's Atmosphere and Ionosphere, 2007, Springer Netherlands, Dordrecht, doi:10.1007/978-94007-0326-1, 2011.

Perkins, F.: Spread F and ionospheric currents, J. Geophys. Res., 78, 218-226, doi:10.1029/JA078i001p00218, 1973.

Saito, A., Iyemori, T., Sugiura, M., Maynard, N. C., Aggson, T. L., Brace, L. H., Takeda, M., and Yamamoto, M.: Conjugate occurrence of the electric field fluctuations in the nighttime midlatitude ionosphere, J. Geophys. Res., 100, 21439, doi:10.1029/95JA01505, 1995.

Shiokawa, K., Otsuka, Y., Ejiri, M. K., Sahai, Y., Kadota, T., Ihara, C., Ogawa, T., Igarashi, K., Miyazaki, S., and Saito, A.: Imaging observations of the equatorward limit of midlatitude traveling ionospheric disturbances, Earth Planet. Space, 54, 57-62, 2002.

Shiokawa, K., Ihara, C., Otsuka, Y., and Ogawa, T.: Statistical study of nighttime medium-scale traveling ionospheric disturbances using midlatitude airglow images, J. Geophys. Res., 108, 1052, doi:10.1029/2002JA009491, 2003a.

Shiokawa, K., Otsuka, Y., Ihara, C., and Ogawa, T.: Ground and satellite observations of nighttime medium-scale traveling ionospheric disturbance at midlatitude, J. Geophys. Res., 108, 1145, doi:10.1029/2002JA009639, 2003b.

Tinsley, B. A. : Field aligned airglow observations of transequatorial bubbles in the tropical F-region, J. Atmos. Terr. Phys., 44, 547557, doi:10.1016/0021-9169(82)90144-1, 1982.

Tinsley, B. A., Christensen, A. B., Bittencourt, J., Gouveia, H., Angreji, P. D., and Takahashi, H.: Excitation of oxygen permitted line emissions in the tropical nightglow, J. Geophys. Res., 78, 1174-1186, doi:10.1029/JA078i007p01174, 1973.

Tsunoda, R. T.: On the coupling of layer instabilities in the nighttime midlatitude ionosphere, J. Geophys. Res., 111, A11304 doi:10.1029/2006JA011630, 2006.

Tsunoda, R. T. and Cosgrove, R. B.: Coupled electrodynamics in the nighttime midlatitude ionosphere, Geophys. Res. Lett., 28, 4171, doi:10.1029/2001GL013245, 2001.

Yokoyama, T. and Hysell, D. L.: A new midlatitude ionosphere electrodynamics coupling model (MIECO): Latitudinal dependence and propagation of medium-scale traveling ionospheric disturbances, Geophys. Res. Lett., 37, L08105, doi:10.1029/2010GL042598, 2010.

Zhou, Q. and Mathews, J. D.: On the physical explanation of the Perkins instability, J. Geophys. Res., 111, A12309, doi:10.1029/2006JA011696, 2006. 